\title{
Nuevo lenguaje, nuevas herramientas, nuevas experiencias: la visita virtual y las etiquetas Navilens en el Museo Arqueológico de Murcia
}

\author{
New language, new tools, new experiences: the virtual visit and the \\ Navilens labels at the Archaeological Museum of Murcia
}

Luis Enrique de Miquel Santed ${ }^{a}$, Raquel Baeza Albaladejob y Teresa Fernández Azorín ${ }^{\mathrm{c}}$

aluisedemiquel@gmail.com, ${ }^{b}$ raquelbaezaalbaladejo@gmail.com ${ }^{\mathrm{C}}$ teresaazorin8@hotmail.com

\begin{abstract}
Resumen
Desde hace algunos años el Museo Arqueológico de Murcia (MAM), consciente de las oportunidades que brinda el uso de las tics para acercar la institución a la sociedad, ha estado planificando y ejecutando diferentes estrategias digitales. Asi nació su equipo dedicado a la realización de nuevas experiencias patrimoniales aplicadas a la didáctica.
\end{abstract}

Actualmente, a través de su visita virtual, pretende introducir el patrimonio arqueológico de la Región de Murcia en todos los hogares. Ésta ofrece la oportunidad no solo de recorrer el espacio libremente, sino también, mejorar la experiencia del usuario a través de una serie de marcadores interactivos.

Además de ser totalmente accesible a través de su página web, esta nueva herramienta está siendo utilizada actualmente de varias maneras. Por el público general, para realizar visitas autónomas desde su casa. Por los colegios u otros colectivos desde sus centros, dirigidos por el personal del museo. Además, se está utilizando en la realización de actividades personalizadas como por ejemplo, la visita-taller realizada con grupos de primaria de Altas Capacidades, en colaboración con la Consejería de Educación de la Comunidad Autónoma de la Región de Murcia.

Por otro lado, el MAM ha apostado por el uso de las "Etiquetas Inteligentes Navilens", una herramienta parecida a las conocidas etiquetas $Q R$ pero mejorada. Su uso ha permitido alojar en ellas más información de la que se incluye en la cartelería convencional: videos, fichas catalográficas, fichas de yacimientos, imágenes, así como la posibilidad de locución de su carteleria. En un futuro se planea incluir documentos de lectura fácil y videos en lengua de signos. Esto favorece la accesibilidad del museo y su apertura a otros públicos que hasta ahora encontraban dificultades en su visita a este tipo de instituciones.

Si se unen estas dos nuevas acciones, el MAM está apostando como institución por ofrecer la posibilidad de una visita totalmente autónoma y accesible que 
Nuevo lenguaje, nuevas herramientas, nuevas experiencias: la visita virtual y las etiquetas Navilens en el Museo Arqueológico de Murcia

permita al usuario, si lo desea, realizar prácticamente una "visita a la carta". Este debe ser el nuevo lenguaje al que deben dirigirse las instituciones museisticas. No se pretende, ni se quiere, sustituir el trabajo humano de las visitas guiadas si no ofrecer nuevas posibilidades complementarias que aporten una mayor experiencia museística

La comunicación en la sociedad ha cambiado y los museos están al servicio de ésta, por tanto, el mensaje y la manera de emitirlo debe cambiar.

Palabras clave: accesibilidad, arqueología, comunicación, digital, Museo.

\footnotetext{
Abstract

For some years now, the Archaeological Museum of Murcia (MAM), aware of the opportunities offered by the use of tics to bring the institution closer to society, has been planning and executing different digital strategies. Thus was born his team dedicated to the realization of new heritage experiences applied to didactics. Thus was born his team dedicated to the realization of new heritage experiences applied to didactics.
}

Currently, through his virtual visit, he intends to introduce the archaeological heritage of the Region of Murcia in all homes. This offers the opportunity not only to move around the space freely, but also to improve the user experience through a series of interactive markers.

In addition to being fully accessible through its website, this new tool is currently being used in various ways. For the general public, to make autonomous visits from their home. By schools or other groups from their centers, directed by museum staff. In addition, it is being used in the realization of personalized activities such as, for example, the visit-workshop carried out with high-ability primary groups, in collaboration with the Consejería de Educación de la Comunidad Autónoma de la Región de Murcia.

On the other hand, MAM has opted for the use of "Etiquetas Inteligentes Navilens", a tool similar to the well-known QR tags but improved. Their use has allowed them to house more information than is included in conventional posters. videos, catalog cards, site files, images, as well as the possibility of announcing their posters. In the future it is planned to include easy-to-read documents and videos in sign language. This favors the accessibility of the museum and its openness to other audiences that until now have encountered difficulties when visiting this type of institution.

If these two new actions are brought together, the MAM is betting as an institution to offer the possibility of a totally autonomous and accessible visit that allows the 
user, if they wish, to carry out practically an "à la carte visit". This should be the new language that museum institutions should address. It is not intended, nor is it intended, to replace the human work of guided tours, but to offer new complementary possibilities that provide a greater museum experience

Communication in society has changed and museums are at its service, therefore, the message and the way it is delivered must change

Keywords: accessibility, archeology, communication, digital, Museum. 
Nuevo lenguaje, nuevas herramientas, nuevas experiencias: la visita virtual y las etiquetas Navilens en el Museo Arqueológico de Murcia

\section{Antecedentes de la didáctica en el MAM}

El Museo Arqueológico de Murcia tiene una larga tradición en la implementación de programas y materiales didácticos para la divulgación de sus contenidos, especialmente en el ámbito educativo.

Ya en los años ochenta se editaron los primeros materiales didácticos. Básicamente eran fichas y cuadernillos, donde se adaptaba la información acerca de la prehistoria y arqueología de la Región de Murcia, a un lenguaje y formato adecuado para los más jóvenes, incluyendo gran cantidad de ilustraciones y algunas actividades rúnico-plásticas para su realización por los jóvenes visitantes.

Sin embargo, el verdadero desarrollo del DEAC del MAM tiene lugar con su reapertura en el año 2007 (tras un paréntesis de casi diez años de obras y reformas en su sede). A partir de este momento se le dota de un equipo estable de monitores-guías para las visitas escolares diarias y las actividades de fines de semana y especiales, y se incrementa exponencialmente los materiales pedagógicos, ahora diversificados por edades y temáticas; que forma que se adaptará a los currículos educativos desde educación infantil hasta el bachillerato.

Empezaron siendo solamente cuadernillos, pero han evolucionado a talleres más completos, aprovechando las posibilidades audiovisuales del Aula didáctica, y, conceptualmente, de la mera divulgación histórica a propuestas más lúdicas (juegos, concursos, gymkanas) y creativas (manualidades, expresión artística) (Fig.1).

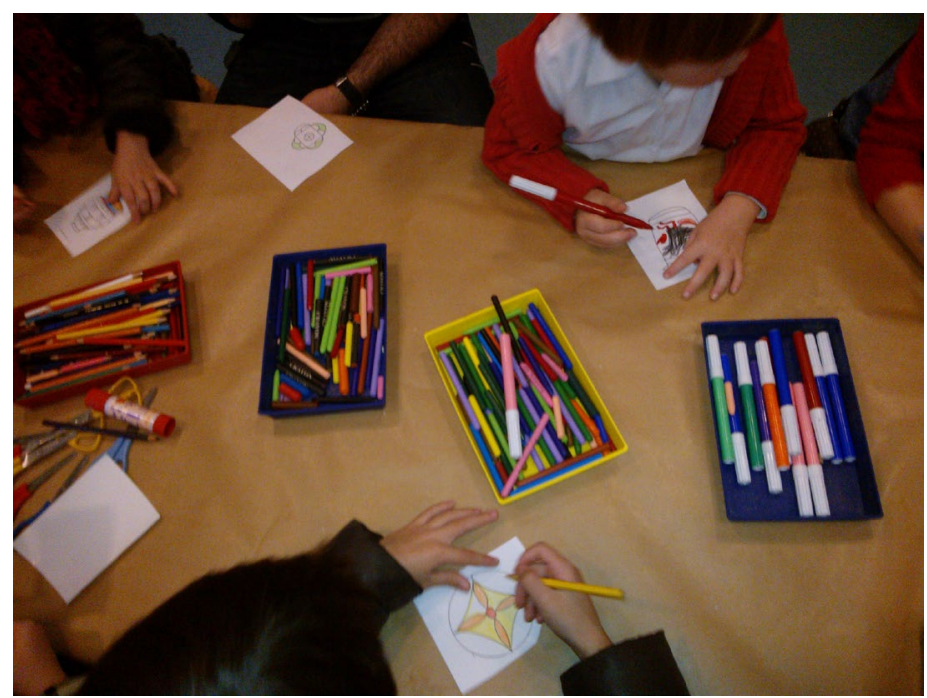

Fuente: Elaboración Propia

Fig. 1 Ejemplo de talleres didácticos

Actualmente, entre los talleres escolares para todos los niveles (bajo el ambicioso objetivo que "ningún niño que visite el museo en todos los años de su formación escolar, repita nunca el 
mismo taller"), los talleres de fines de semana, los monográficos para momentos especiales del año (talleres de verano, navidades, semana de la mujer....) y los asociados a las numerosas exposiciones temporales que vamos desarrollando cada año, tenemos en el Museo Arqueológico de Murcia, diseñados y elaborados, unos doscientos cincuenta talleres didácticos, convencionales.

\section{Accesibilidad y difusión la aplicación de las tecnologías en los museos}

El desarrollo social siempre ha avanzado a la par que el desarrollo tecnológico, en un proceso que se retroalimenta, la sociedad desarrolla la tecnología y la tecnología hace avanzar a la sociedad. Podemos incluir a los museos como elemento de este suceso en el sentido que nos indica María del Rocío Acha Barral (2006) cuando dice que los museos y centros culturales públicos o privados son portadores de cultura, pero también son agentes de cambio social.

Pero actualmente, la velocidad del desarrollo ha provocado un aumento cualitativo de las tecnologías sobre todo las digitales, permitiendo la creación de nuevos entornos visuales que están convirtiendo a meros visitantes de museos en usuarios que interactúan con los materiales y la información de un modo más autónomo. Sin dejar de lado la figura del guía del museo, todas estas tecnologías suponen un complemento a las visitas guiadas.

El concepto de nuevas tecnologías puede presentar diacronías porque varía a lo largo del tiempo, haciendo referencias a los distintos desarrollos técnicos en continua reciprocidad con el desarrollo social; por este motivo entendemos que la tecnología que se denominó como "nueva" hace una década actualmente ha perdido esa condición, aunque se entiende el uso del vocablo por convención social. En nuestro caso nos referiremos indistintamente a nuevas tecnologías, tecnologías avanzadas o tecnologías sin más.

Aunque tradicionalmente el origen del desarrollo tecnológico provenía del ámbito militar (internet, GPS, drones, etc.) en la actualidad la investigación y el desarrollo sufren un proceso de diversificación proporcionando nuevos avances a partir de la industria cinematográfica o del ámbito académico especializado, como sucede con los proyectos de $\mathrm{I}+\mathrm{D}$ en centros como el Instituto Tecnológico de Massachusetts (MIT, Cambridge). En los museos se comenzó entre finales de los años 80 e inicios de los 90 con los primeros ordenadores y aunque el desarrollo de las tecnologías no llegó de forma inmediata el siglo XXI supuso una apertura que va a más en esta modalidad e incluso a día de hoy la mayoría de estas instituciones están presentes en las redes a través de la web 2.0 o de redes sociales transformando el contacto con los públicos (San Martín y Cordero, 2018).

La implementación de estas tecnologías en la sociedad, y más concretamente en el ámbito museístico que nos ocupa, depende de una labor por parte de administraciones o entidades que propongan o lleven a cabo la instalación de hardware o software avanzado, adaptado a las necesidades y deseos de los visitantes. En la mayoría de casos se ha implementado en los museos a través de la tecnología audiovisual, desde la instalación de pantallas o proyectores para la reproducción de contenidos específicos, hasta las tecnologías táctiles que proporcionan una 
Nuevo lenguaje, nuevas herramientas, nuevas experiencias: la visita virtual y las etiquetas Navilens en el

interacción más fluida; en todos los casos el objetivo es facilitar el acceso a la información de manera inclusiva, ágil y atractiva.

Esta implementación tecnológica permite también la creación de entornos virtuales que suponen una mejora para la economía del espacio, para la diversificación de contenidos y para la síntesis de la información. Gracias a la incorporación de nuevas técnicas audiovisuales el usuario estimula su curiosidad o su interés por la historia a través de productos derivados del 3D como el videomapping, visita virtual, audioguías restauración o anastilosis virtual, modelos digitales del terreno, scanner laser o fotogrametría y sus derivados en modelos tridimensionales, impresiones 3D (incorpora el sentido del tacto y se puede usar para la accesibilidad para personas invidentes o contactos para niños más pequeños, puzles, dioramas).

\section{Objetivos}

Teniendo en cuenta lo anteriormente expuesto, podemos hacernos una idea de cómo la accesibilidad y la difusión se ven facilitadas por la implementación del desarrollo tecnológico en los museos, estableciendo nuevos paradigmas que resultan aprovechables y mejoran la experiencia e interactuación del usuario con los contenidos de los museos.

Con todo, desde el Museo Arqueológico de Murcia queremos exponer las dos nuevas tecnologías que recientemente se han implementado en el museo: Las Etiquetas Navilens y la Visita Virtual.

\section{Etiquetas Navilens}

En febrero de 2019 salía en prensa, un proyecto en el que el personal del Museo Arqueológico de Murcia llevaba varios meses trabajando para mejorar las visitas de todo tipo de usuarios a través de la tecnología. Consistía en la implantación de unas etiquetas inteligentes desarrolladas por la empresa murciana Neosistec denominadas "Etiquetas Navilens". Esta es una tecnología destinada a ser la nueva señalética digital "diseñada para todos", fomentando la filosofía inclusiva (Fig.2).

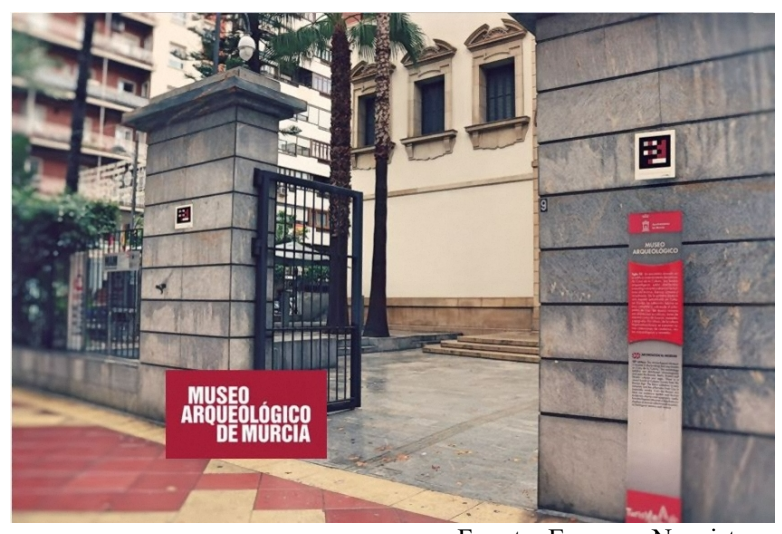

Fuente: Empresa Neosistec

Fig. 2 Entrada del MAM con la Etiqueta Navilens 
Estos marcadores consisten en un sistema de señalización digital universal que no requiere de hardware y/o dispositivos específicos más allá del propio dispositivo móvil del usuario. Así estas etiquetas son detectadas de forma ultra rápida, a larga distancia y en gran ángulo por los dispositivos móviles de los usuarios por lo que no es necesario enfocar. Además, el dispositivo puede detectar los marcadores mientras el usuario va caminando y detecta múltiples marcadores simultáneamente.

Son etiquetas similares a lo que actualmente es una $\mathrm{QR}$, con la particularidad de incorporar el color, lectura segura con comprobación de errores y muy alta densidad. Asimismo, esta iniciativa se presentó en el Congreso Internacional "El museo para todas las personas: arte, accesibilidad e inclusión social”, en abril de 2019 en Madrid.

Este nuevo concepto de señalética creada en un principio para los usuarios con discapacidad visual ha evolucionado constantemente desde su creación gracias al esfuerzo de la dirección del museo y la propia empresa, y así, se han podido incorporar experiencias adaptadas a un público general, con discapacidad sonora y existe proyectos de incorporar contenidos para las personas con discapacidad cognitiva, o público infantil.

Actualmente, existen dos aplicaciones para el reconocimiento de etiquetas, una orientada a usuarios con discapacidad visual y otra para un público sin discapacidad visual.

La primera, Navilens, es la aplicación para el primer grupo de personas. Ésta utiliza el idioma del dispositivo del usuario, por lo que no es necesario cambiar el idioma de la App; además locuta la información y ofrece a la persona una orientación espacial debido a que brinda de manera sonora la distancia que existe desde el dispositivo a la etiqueta orientando al usuario con instrucciones de movimiento.

La segunda, Navilens Go!, fue desarrollada entre el equipo del MAM y la empresa para poder llegar a un público más amplio. Ofrece información ampliada sobre los elementos del museo (con videos, fotos, fichas técnicas...), así como información locutada.

Actualmente, en el museo existen unas doscientas cincuenta etiquetas, distribuidas en tres niveles de lectura. Sin embargo, en todas se han implementado distintos recursos como PDFs, imágenes y videos. Se pueden diferenciar a simple vista, pues cuanto más grande es la etiqueta los contenidos que ofrecen son más generales.

- Un primer nivel, con información sobre la sala. En este nivel, las etiquetas se colocan normalmente sobre la cartelería general. Los contenidos están relacionados con el tema general de la sala, aportando más información sobre ella. Por ejemplo sobre el hábitat de los íberos.

- Un segundo nivel con información sobre la vitrina. Todas estas instalaciones museográficas del museo cuentan con una de las etiqueta Navilens. En este caso, los contenidos están relacionados con el tema de la vitrina y las piezas en ella expuestas. Por ejemplo sobre la cerámica pintada íbera. 
Nuevo lenguaje, nuevas herramientas, nuevas experiencias: la visita virtual y las etiquetas Navilens en el Museo Arqueológico de Murcia

- Un tercer nivel de información sobre la pieza. De las 2500 piezas expuestas en el museo se han seleccionado 100 para profundizar sobre ellas a través de las etiquetas Navilens. Entre los contenidos se pueden observar fotos detalle de las piezas, ficha técnica o una somera explicación del yacimiento al que pertenece (Fig.3).

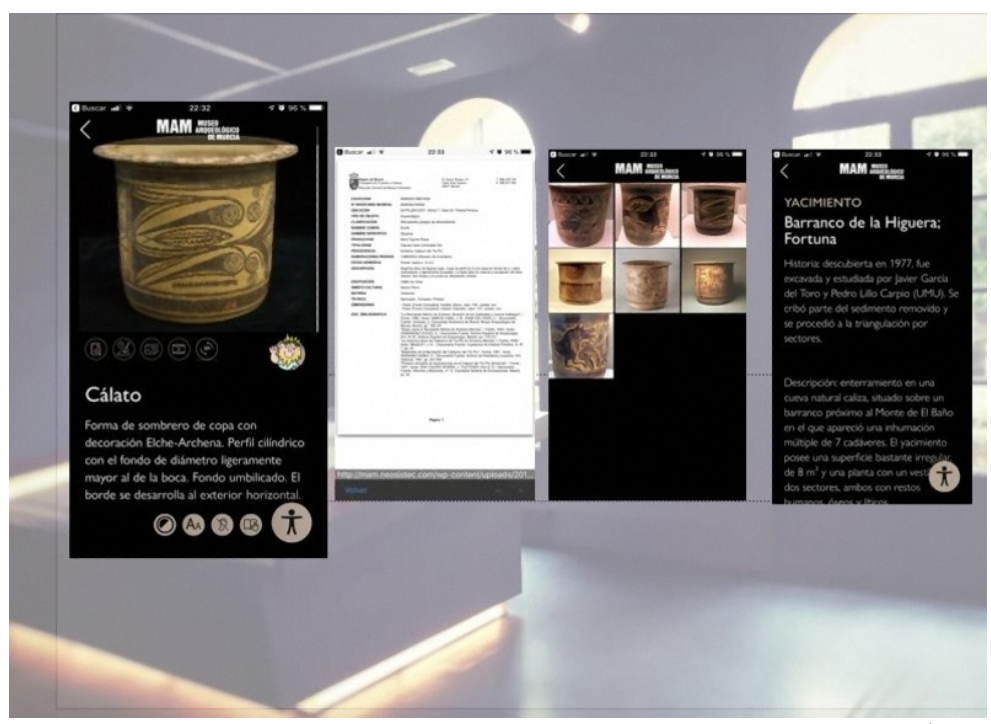

Fuente: Empresa Neosistec

Fig.3 Distintas pantallas de la APP NAvilens Go con distintos tipos de información complementaria

En el momento en el que se estaba trabajando en la implementación de videos en lengua de signos, información en lectura fácil y pictogramas, además de contenidos adaptadas al público infantil y posibilidad de realizar recorridos, irrumpió en nuestro país el COVID-19, el confinamiento, y las lógicas medidas de higiene y de distanciamiento social que trastocó toda la planificación de proyectos y actividades de 2020 cambiando las prioridades de la institución.

\section{Visita Virtual}

Tras la incorporación de las etiquetas Navilens en 2019, el siguiente proyecto en materia de Tics en el Museo Arqueológico de Murcia, fue la realización de una visita virtual. En ésta se estaba trabajando durante el último trimestre de 2019, para poder presentarla en 2020, cuando llegó el COVID-19.

Es evidente que éste ha transformado la vida de las instituciones, su manera de relacionarse con el público y éste con las colecciones. Parece que en un futuro cercano no habrá lugar para salas abarrotadas. Sin embargo, el equipo del museo piensa también que es un momento de oportunidades. En el mundo post-Covid19, los museos dejarán de ser objetivo del turismo masivo y, los indicadores de éxito serán menos cuantitativos y más cualitativos, liberándose por otro lado de la tiranía de las cifras. 
Pensamos que este es el momento de ahondar en la sostenibilidad de los museos, para la mejor preservación de los bienes y, mejorar la calidad de la experiencia de los ciudadanos.

En este sentido, la visita virtual, al menos la del MAM, es un tipo de visita que se puede realizar a través de la pantalla de tu ordenador o dispositivo electrónico. De esta manera, se pueden recorrer todas las salas de esta institución de manera interactiva e intuitiva.

Además esta herramienta nos ha permito reinventar la manera de ver el museo y ha puesto en funcionamiento varias experiencias de visita: la general, con guía y con actividades extracurriculares.

\subsection{La Visita Virtual General}

Así, esta herramienta está realizada a través de Matterport, por medio de lo que se denomina gemelo digital, es decir una copia digital de un lugar u objeto en el mundo real.

Accesible a todo el mundo a través de la página web del museo (Fig.4) la vista virtual que ofrece el MAM permite los siguientes usos: que el visitante planifique la visita al museo desde casa y, que las personas que no puedan acudir al museo real, sobre todo ahora con la situación actual de crisis sanitaria, disfruten del mismo teniendo acceso a la versión virtual.

Unas características que la hace diferente del resto de visitas virtuales son los marcadores. No los marcadores en sí, pues muchas visitas virtuales tienen diferentes tipos de marcadores sino que los de esta visita virtual están conectados con los de la App Navilens Go.

Esto permite que los usuarios de la visita virtual tengan el acceso a la misma información y experiencias que los visitantes del museo que están utilizando la App.

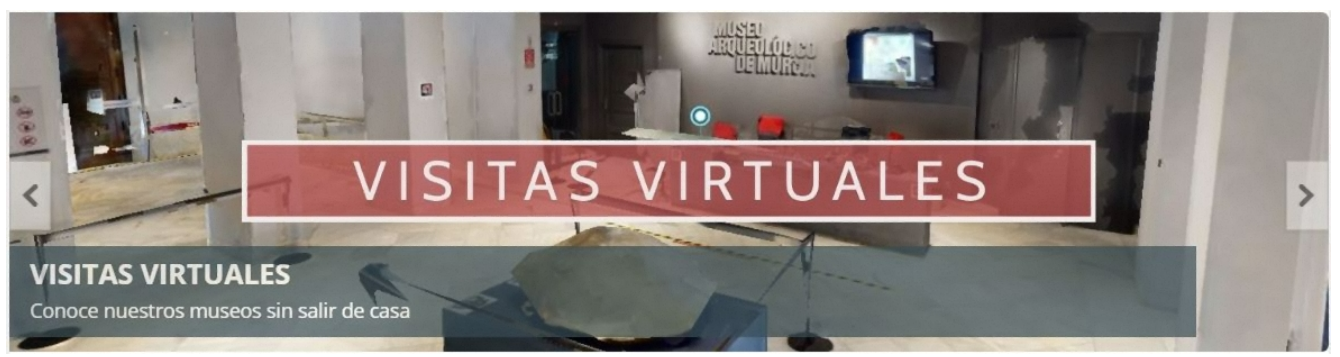

Fuente: Página Web del museo

Fig. 4 Portada de la visita virtual en la Página Web del museo

\subsection{La Visita Virtual Guías}

La conjunción de estas nuevas tecnologías ("etiquetas inteligentes" y "visita virtual”), con la atesorada tradición de visitas guiadas presenciales del MAM desde el año 2007, ha permitido dar respuesta a la caída generalizada de presencia de grupos de visitantes al museo.

Atendiendo a las circunstancias que hoy nos afectan, desde el MAM se ha planteado la posibilidad de seguir siendo un referente tanto a nivel educativo como cultural. Es por ello que 
Nuevo lenguaje, nuevas herramientas, nuevas experiencias: la visita virtual y las etiquetas Navilens en el

se ha puesto en marcha el programa "El Museo al cole" y las "Visitas telemáticas dominicales al MAM" (Fig.5).

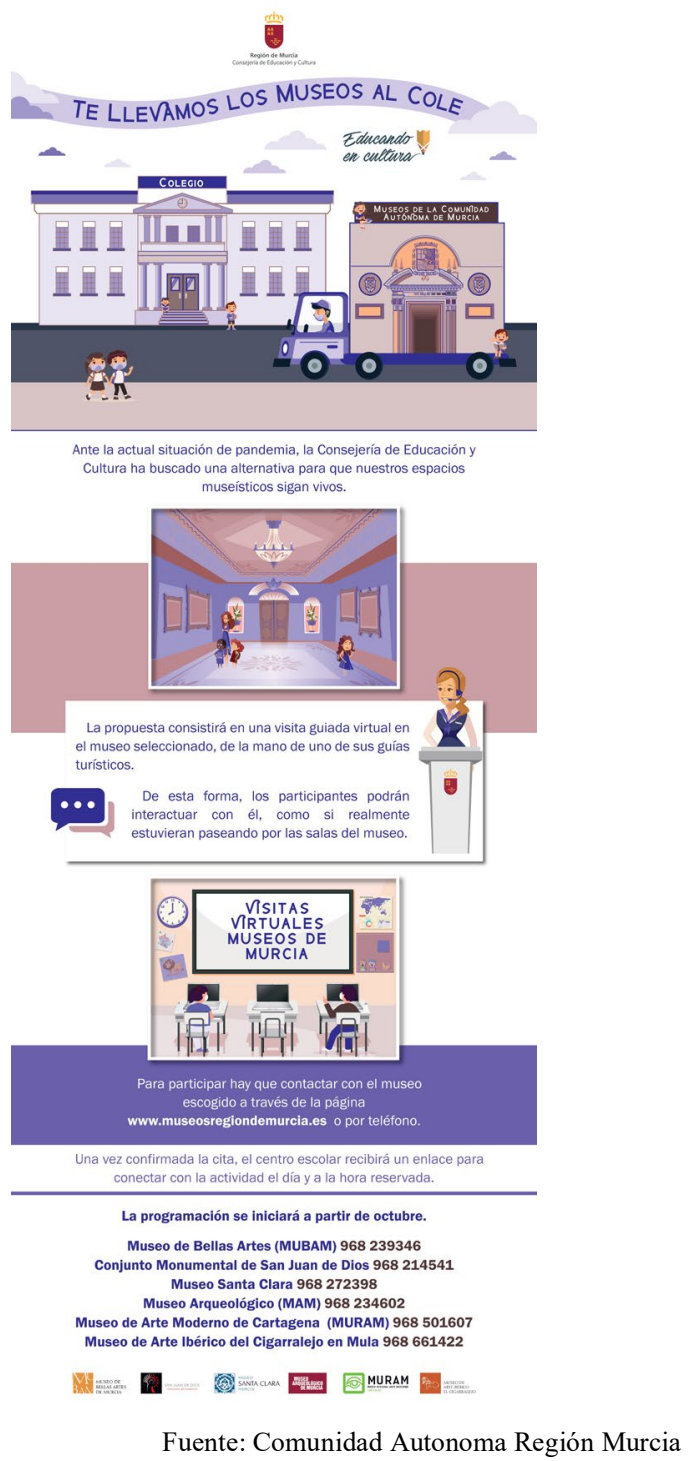

Fig. 5 Cartel "Te llevamos el museo al cole"

Cuando hablamos de visitas telemáticas, no hacemos referencia en nuestro caso, a facilitar la posibilidad que el "visitante virtual" discurra por las distintas salas, a su gusto y antojo, sino a un itinerario completo donde la "visita concertada virtual" tiene el acompañamiento de las propias guías del museo, que van llevando al visitante por las distintas salas y parándose en aquellos espacios más interesantes o en donde surjan dudas a lo largo de la misma. Es decir, tener una visita tradicional, pero en la que ninguno de los sujetos que participan está "in situ" en el museo, sino conectados desde sus colegios o casas. 
No se trata tampoco de un video pregrabado inamovible, sino de una verdadera retransmisión en streaming, pero donde en vez de tener la imagen fija del conferenciante o guía, nos movemos dinámicamente por las salas correspondientes a la temática que se desarrolle.

Se pueden hacer preguntas al guía y éste interactúa con los participantes en la "visita virtual" en tiempo real. Asimismo, el taller se completa con pequeñas actividades didácticas (manualidades, juegos...) que presentan las guías y que se pueden enviar por el correo de contacto a los participantes, para que las completen en el aula o desde sus casas.

Con esta pequeña propuesta se pretende seguir apoyando la educación, y específicamente a la educación patrimonial, como una colaboración con las actividades extraescolares de los docentes, en estos momentos tan comprometidos.

Pese a que siempre defenderemos la visita presencial al museo como la mejor experiencia cultural y lúdica para los ciudadanos, grandes o pequeños, estamos valorando que este tipo de visitas se pueda mantener sin fecha de caducidad, ante la constatación que algunos centros escolares y público en general que viven lejos del museo arqueológico, han tenido siempre muy difícil la posibilidad de acercarse físicamente al centro y podemos ahora ofrecerles una nueva posibilidad.

En el momento actual, cuando llevamos escasamente tres meses de esta nueva propuesta educativa, estamos muy satisfechos por la respuesta: una media de cincuenta grupos al mes visitan virtualmente el MAM (casi tantos como lo hacían presencialmente antes de los confinamientos) y aspiramos a un incremento en los próximos meses hasta que puedan volver a asistir normalmente a nuestras salas. Actualmente ante el éxito de las visitas virtuales desde la dirección del museo se ha tomado la decisión de ampliar esta oferta a los domingos por la mañana. (Fig. 6). 


\title{
Más de 5.000 escolares visitarán los museos de forma virtual este curso
}

\author{
Los alumnos pueden descubrir estos espacios a través de un \\ recorrido 'online' guiado que permite la interacción
}

\begin{abstract}
LA VERDAD
MURCIA

$f y \cdots$ ด

Lunes, 25 enero 2021, 10:18

Este curso, el acceso de los escolares a los museos regionales está siendo virtual gracias al programa 'Te llevamos los museos al cole', de la Consejería de Educación y Cultura, que permite mantener la aproximación de los estudiantes a estos espacios sin desplazamientos y cumpliendo las pautas sanitarias del Covid-19.

Desde su inicio el pasado 23 de noviembre, e incluyendo las reservas hasta la fecha, 267 grupos con 5.607 escolares habrán visitado estos espacios cuando concluya el curso 2020-2021. Un total de 59 grupos con 1.239 alumnos ya han participado en las visitas virtuales desde sus aulas, y desde ahora hasta final de curso tienen programada su asistencia 208 grupos con 4.368 escolares.
\end{abstract}

Fuente: Periódico La Verdad.

Fig. 6 Noticia en prensa sobre la visita virtual

Para una más extensa información sobre este tipo de visitas y las temáticas (cambiantes cada mes) que abordamos en ellas remitimos a la comunicación presentada al EDUNOVATIC 2020. V CONGRESO VIRTUAL INTERNACIONAL DE EDUCACIÓN, INNOVACIÓN Y TIC, por nuestro propio equipo de educadoras del Museo Arqueológico de Murcia (http://www.edunovatic.org/actas).

\subsection{La Visita Virtual Altas Capacidades}

En el año 2019 llegó al museo una propuesta desde la Consejería de Educación y Cultura, dentro del ámbito de atención a la Atención a la Diversidad del Equipo Específico de Altas Capacidades. Nos solicitaban la creación de un proyecto que se pudiera realizar en el Museo Arqueológico de Murcia para niños de Altas Capacidades, con edades comprendidas entre 12 y 13 años. Este proyecto debía realizarse con un modelo metodológico conocido como "Enriquecimiento", consistente en ofrecer tareas, actividades y (retos) problemas, cuyas soluciones exigen de habilidades socio-cognitivas, tales como: razonamiento, planificación, análisis, síntesis, deducción, creatividad, toma de decisiones o trabajo cooperativo, para la 
resolución de problemas. Uno de los objetivos generales que se debían cubrir era el de desarrollar aptitudes intelectuales, que ayuden a mejorar el pensamiento crítico, científico y lógico, necesario para el conocimiento eficiente y el aprendizaje autónomo, para ello nos centramos en alentar la curiosidad por el pasado y por la historia de nuestro grupo, prestando especial atención en que entendieran los procesos, avances y cambios de la sociedad con el paso del tiempo. Otro de los puntos que tratamos en el taller fue transmitirles la complejidad y la importancia de la adecuación de los contenidos para obtener un resultado exitoso a la hora de que mostrar y difundir para que el resultado sea deseado, además de aprender el significado de la difusión y generar un modo propio de realizarla.

Para fomentar el pensamiento creativo y su pertinente aplicación a la vida cotidiana lo que trabajamos en el taller fue el uso de las nuevas tecnologías de las que disponíamos, como las etiquetas Navilens, las bases de datos de materiales arqueológicos FORO, las reproducciones en $3 \mathrm{D}$, incorporando en este segundo año la visita virtual la Visita Virtual. Integramos como herramientas de trabajo sus Smartphones y sus Tablet que les servían como soporte en la búsqueda de información y la obtención de ideas para sus respectivos trabajos, de este modo pudieron incentivar el carácter resolutivo aprovechando los medios a su alcance para la elaboración de las tareas propuestas.

Por último y para incentivar el desarrollo de habilidades autónomas de gestión del pensamiento y procesos cognitivos, trabajamos con la creación de discursos inclusivos, para mostrarles que sin pérdida de rigor científico puede generarse un guion comprensible para todos. Se incentivó el trabajo en grupo y se fomentó el respeto a la escucha de los demás para aprovechar el aprendizaje grupal e individual.

El proyecto denominado "Jóvenes enganchados al patrimonio", se desarrollaba en el museo, donde el grupo conocería no solo la parte expositiva de este, sino que entraría en las mismas entrañas del edificio visitando los fondos, el área de restauración, el almacén visitable, las oficinas, etc. para entender todo el desarrollo de trabajos que conlleva esta institución. Una vez que estuvieran familiarizados con el lugar podrían desarrollar su propio discurso adaptado a su edad y a sus congéneres para exponer su experiencia desde diferentes líneas de trabajo, la museografía, la documentación y la difusión.

En este segundo año de talleres y debido a la situación actual provocada por el COVID-19, tuvimos que realizar una adaptación del proyecto para el ámbito virtual, ya que era imposible realizar las sesiones del taller de forma presencial. Gracias a las tecnologías desarrolladas en el museo disponíamos de herramientas que nos permitieron realizar esta adaptación, la visita virtual, las Etiquetas Navilens y los vídeos generados sobre temáticas y exposiciones realizadas; además de toda la información que a través de internet podían consultar sobre los lugares de donde procedían la mayoría de los objetos, épocas y culturas que se exponen en el museo. Dando un giro a la propuesta inicial de este trabajo para seguir manteniendo el cumplimiento de los objetivos se desarrolló con el grupo la creación de una visita de modo virtual, serían los propios alumnos quiénes realizarían la Visita Virtual, aprendiendo a manejarse con esta herramienta, diseñarían sus paradas, dando contenido a cada una de ellas y generando un discurso que 
Nuevo lenguaje, nuevas herramientas, nuevas experiencias: la visita virtual y las etiquetas Navilens en el Museo Arqueológico de Murcia

acompañaría esta visita en todo momento, se sirvieron de la cartelería, del contenido de las vitrinas, ellos seleccionarían las piezas a mostrar y las etiquetas incluidas en la visita proporcionarían no solo la información para la preparación además podrían incluirlas en su visita.

De este modo y gracias a la Visita Virtual hemos podido solventar el problema de los talleres no presenciales, además de mostrar a los niñ@s una nueva forma de ver el museo y hemos comprobado que esta experiencia les sirve como invitación a la visita personal al museo para poder ver en vivo lo que han trabajado en virtual.

\section{Conclusiones: Donde hemos llegado y hacia donde nos dirigimos}

Para concluir, queremos destacar que lo cierto es que el Covid-19 ha cambiado la manera que tenía la institución de relacionarse con el público y viceversa, pero también ha posibilitado nuevos canales de comunicación y llegar a un visitante que antes no asistía al museo.

Gracias a su Departamentos de Educación y Acción Cultural, el Museo Arqueológico de Murcia tiene a un público escolar fidelizado que viene todos los años a la institución para realizar distintas visitas con sus correspondientes talleres, pero estos grupos solían ser de la ciudad de Murcia, alrededores, y colegios que pueden realizar actividades fuera del aula sin que suponga un gasto excesivo de recursos y logística. Para ellos, tanto la visita virtual como las etiquetas Navilens son "sólo" herramientas para seguir con su programación habitual.

Pero hay otro tipo de grupos escolares que no podían asistir al museo con tanta asiduidad, para ellos esta visita virtual guiada supone una oportunidad de enriquecer sus actividades. Un tipo de visitante por el que se debe seguir manteniendo este tipo de visitas en un futuro, para que el museo cumpla de una manera más eficaz con su función social ya que al final, como se ha demostrado, estas herramientas permiten llegar a un público más amplio.

Esto ha llevado al equipo de museo a plantearse diferentes estrategias de difusión de estos instrumentos, para que la sociedad pueda conocer las diferentes herramientas con las que cuenta en su visita al museo.

Un ejemplo, los días "especiales" en los que la oferta cultural se amplia como el día de los museos o el de la mujer trabajadora. En estos días, los medios de comunicación más convencionales como los periódicos dedican más espacio a las iniciativas culturales y existe una mayor movilización entre la sociedad para realizar diferentes actividades culturales.

Pero no debemos olvidar, a las redes sociales como Facebook e Instagram y Tik Tok donde ya se esta observando la facilidad de llegar a un público más amplío y que es posible que no se encuentre en la misma localidad que la institución museística que sigue. La figura del community manager es imprescindible en esta estrategia de difusión.

Pero para poder continuar con estas tecnologías y que se puedan mantener en el tiempo, es necesario su desarrollo y mantenimiento tanto con financiacion económica como con recursos humanos para su promoción y aprovechamiento. 
La toma de conciencia de las instituciones sobre la situacion actual ha permitido que éstas herramientas sean posibles gracias a una mayor financiacion. Pese a lo que pueda parecer no se elimina el factor humano sino que incluso lo pueden potenciar.

Sin embargo, es cierto que es más difícil que estas herramientas lleguen a los pequeños museos que cuentan con un presupuesto modesto, por lo que desde el equipo del museo se considera interesante la creación de lineas de subvenciones o financiación relacionadas con la implantación de estas tecnologías, que tan útiles han demostrado ser en estos tiempos de resilencia para todos.

Para finalizar, desde el equipo de museo queremos exponer el futuro más cercano de esta Visita Virtual y las Etiquetas Navilens: crear actividades didácticas y la posibilidad de jugar con las etiquetas y enlazar directamente con juegos.

\section{Referencias}

ACHA BARRAL, M. D. R. (2006). Dialéctica y nuevas tecnologías de la información en los museos. En La tecnología y la comunicación museística. Murcia: Comunidad Autónoma de la Región de Murcia.

FERNÁNDEZ CABADA T.M., PÉREZ TURPÍN M.J. (2015): “Cuéntame y lo olvidare. Muéstrame y lo recordare. Involucrame y lo entenderé: experiencias didácticas en el Museo Arqueológico de Murcia”, Verdolay: Revista del Museo Arqueológico de Murcia, $\mathrm{n}^{\circ}$ 14, p. 349-361.

La Verdad. Más de 5.000 escolares visitarán los museos de forma virtual este curso https://www.laverdad.es/culturas/5000-escolares-visitaran-20210125011941-ntvo.html > [Consulta: 25 de febrero de 2021]

La Verdad: Tecnología murciana para unos museos regionales más accesibles https://www.laverdad.es/murcia/tecnologia-murciana-accesibles-20190215133326-nt.html [Consulta: 25 de febrero de 2021]

MIQUEL SANTED L.E. (2009) “El Museo Arqueológico de Murcia. Un proyecto de museo para la Murcia del siglo XXI". Museo, no 14, p. 289-311.

SAN MARTÍN GARCÍA B. X.; CORDERO COELLAR E. V. (2018): "Los Museos y las Tecnologías de la Comunicación en Cuenca". Revista Killkana Sociales. Vol. 2, No. 3, p. 171-178. 